\title{
Successful Treatment with a High-dose Rifampin-containing Regimen for Pulmonary Tuberculosis with a Disputed rpoB Mutation
}

\author{
Dae Hyun Jeong ${ }^{1}$, Ye Won Kang ${ }^{1}$, Jin Young Kim ${ }^{1}$, Ji Soo Han ${ }^{1}$, \\ Kyung-Wook $\mathrm{Jo}^{2}$ and Tae Sun Shim ${ }^{2}$
}

\begin{abstract}
:
Mutations in the rроB gene of Mycobacterium tuberculosis can result in resistance to rifampin. Among various mutations in the rров gene, some known as disputed rроB mutations can cause low-level rifampin resistance. It has been suggested that a high-dose rifampin $(20 \mathrm{mg} / \mathrm{kg})$-based regimen might be effective in treating tuberculosis (TB) caused by M. tuberculosis with disputed rpoB mutations exhibiting low-level resistance. We herein report the first two cases of pulmonary TB caused by M. tuberculosis with a disputed rpoB mutation (CTG511CCG) that showed successful treatment outcomes with a high-dose rifampin-based regimen.
\end{abstract}

Key words: tuberculosis, disputed mutation, high-dose rifampin, $r p o B$ gene

(Intern Med 57: 3281-3284, 2018)

(DOI: 10.2169/internalmedicine.9571-17)

\section{Introduction}

Resistance to rifampin (RIF) is a key determinant of treatment failure for patients with tuberculosis (TB) (1). Mutations in the 81-bp region of the $r p o B$ gene, known as the RIF-resistance-determining region (RRDR), have been detected in almost all RIF-resistant Mycobacterium tuberculosis strains. The degree of resistance among the mutations in the RRDR is not equal. For example, some RRDR mutations known as disputed $r p o B$ mutations can cause low-level resistance to $\operatorname{RIF}(2,3)$. Although it has been suggested that high-dose RIF (20 mg/kg) is likely to overcome this lowlevel resistance (4), no studies have been conducted to explore the efficacy of a high-dose RIF-based regimen for disputed mutations thus far.

We herein report two cases of pulmonary TB caused by M. tuberculosis strains with disputed rpoB mutations successfully treated with a high-dose RIF-based regimen.

\section{Case Reports}

\section{Case 1}

A 60-year-old man presented at our hospital with right chest pain persistent for 1 month. He had received treatment for pulmonary TB approximately 40 years earlier. He was again treated for phenotypic pan-susceptible pulmonary TB for 7 months with a standard four-drug regimen [isoniazid (INH) $300 \mathrm{mg}$, RIF $600 \mathrm{mg}$, ethambutol (EMB) 1,000 mg and pyrazinamide (PZA) 1,500 mg], which had been completed 4 months before his most recent presentation. He had no cavitation on initial chest radiograph, and a culture turned negative after two months of treatment. Due to liver enzyme elevation, the drugs were switched to less hepatotoxic ones transiently, so the treatment duration was extended to seven months. A genotypic drug susceptibility test (DST) was not performed at that time. Although the treatment was successful, chest X-ray (CXR) at the completion of treatment showed parenchymal destructive lesions with fi-

${ }^{1}$ Department of Internal Medicine, University of Ulsan College of Medicine, Asan Medical Center, Korea and ${ }^{2}$ Division of Pulmonary \& Critical Care Medicine, University of Ulsan College of Medicine, Asan Medical Center, Korea 


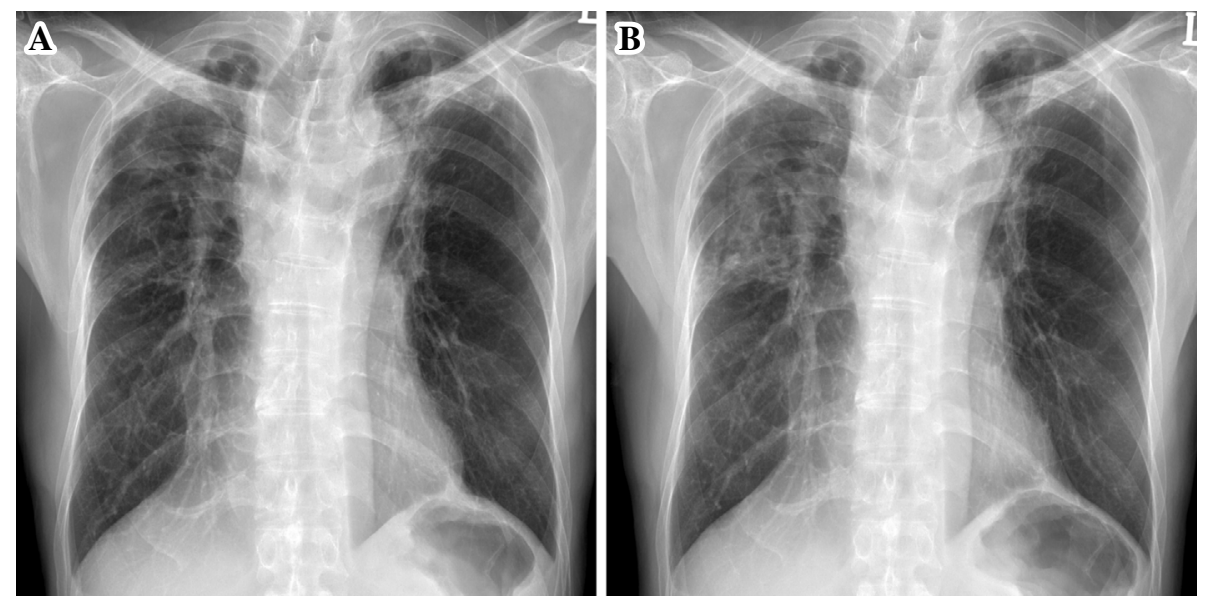

Figure 1. (A) Chest X-ray taken four months earlier showing parenchymal destruction and fibrotic lesions in the bilateral upper lungs. (B) Chest X-ray showing newly developed infiltrative lesions in the right upper lung field.

brotic changes in both upper lungs (Fig. 1A). A physical examination revealed that the patient was alert and not in distress. Routine blood test findings were within the normal ranges. The patient was negative for antibody to human immunodeficiency virus. His vital signs included a temperature of $36.1^{\circ} \mathrm{C}$, blood pressure of $120 / 70 \mathrm{mmHg}$, a heart rate of 73 beats per minute (with a regular rhythm) and a respiratory rate of 20 breaths per minute. Auscultation revealed decreased breath sounds in the anterior chest of both upper lung fields.

Compared to CXR taken four months earlier, his current CXR showed increased opacity on the right upper lung field without cavitation (Fig. 1B). Smears and cultures of multiple sputum specimens were both positive for acid-fast bacilli (AFB), which was confirmed as M. tuberculosis complex by Ziehl-Neelsen staining and a polymerase chain reaction (PCR) assay using a Seeplex tuberculosis detection kit (Seegen, Seoul, Korea). Based on these data, the patient was diagnosed with recurrent pulmonary TB. Treatment was initiated with INH, RIF (600 mg/day), EMB and PZA. The GenoType ${ }^{\circledR}$ MTBDRplus assay (Hain Lifescience, Nehren, Germany) was performed at our center according to the manufacturer's instructions. A phenotypic DST was performed using the absolute concentration method on Löwenstein-Jensen medium at the Korean Institute of Tuberculosis, which is the Supranational Reference Laboratory in South Korea. Wells contained the following critical concentrations of anti-TB drugs: $0.2 \mu \mathrm{g} / \mathrm{mL}$ of INH and $40 \mu \mathrm{g} / \mathrm{mL}$ of RIF. Growth exceeding that of the control wells by $>1 \%$ was considered to indicate drug resistance.

The GenoType ${ }^{\circledR}$ MTBDRplus assay revealed susceptibility to INH and resistance to RIF. Since the $r p o B$ band pattern was suspected of being a disputed mutation (due to the loss of the wild-type probe 2 without hybridization of the mutation probes), DNA sequencing of a 501-bp fragment of the rроB gene was performed using a PCR-direct sequencing system (Cosmo, Seoul, South Korea). The primers were synthesized by JieLi Bio (Hong Kong, China). Sequencing data were assembled and analyzed using the BioEdit software program (Isis Pharmaceuticals, Carlsbad, USA). The rpoB mutations were determined via a comparison with those of H37Rv from the GenBank database (http://www.ncbi.nih.gov /gene), and the CTG511CCG (L511P) mutation of rpoB gene was subsequently identified. Based on this result, the dosage of RIF was gradually increased to a high dosage (20 $\mathrm{mg} / \mathrm{kg}, 1,200 \mathrm{mg} /$ day) for 2 weeks.

After five weeks of treatment, the phenotypic DST indicated that the M. tuberculosis isolate was susceptible to all anti-TB drugs tested, including RIF. Therefore, we decided to maintain the treatment regimen including high-dose RIF. The patient experienced a febrile sensation and easy fatigability five weeks after starting the high-dose RIF regiment. The dose was reduced to $900 \mathrm{mg}$, but the symptoms were not resolved. After 1 week, the dose was increased to 1,200 $\mathrm{mg}$ again, and the subjective symptoms improved spontaneously without any serious adverse events. After two months of treatment, sputum culture for $M$. tuberculosis was negative, and INH and high-dose RIF were maintained thereafter for nine months. Upon completion of treatment, CXR showed an improvement in the lung lesions. The patient has been followed up for 27 months since treatment completion with no recurrence.

\section{Case 2}

A 58-year old man was referred to our hospital with a chief complaint of cough lasting for 3 months. He did not have any history of anti-TB medication. On a physical examination, the patient was alert, and his vital signs were stable. Antibody to human immunodeficiency virus was negative.

CXR showed nodular and increased peribronchial opacity in the right upper lobe without cavitation (Fig. 2A). Multiple sputum smears were positive for $\mathrm{AFB}$, and cultures from both solid and liquid media revealed $M$. tuberculosis. A regimen including INH, RIF (600 mg/day), EMB and PZA was begun. Three weeks later, a GenoType ${ }^{\circledR}$ MTBDRplus assay 


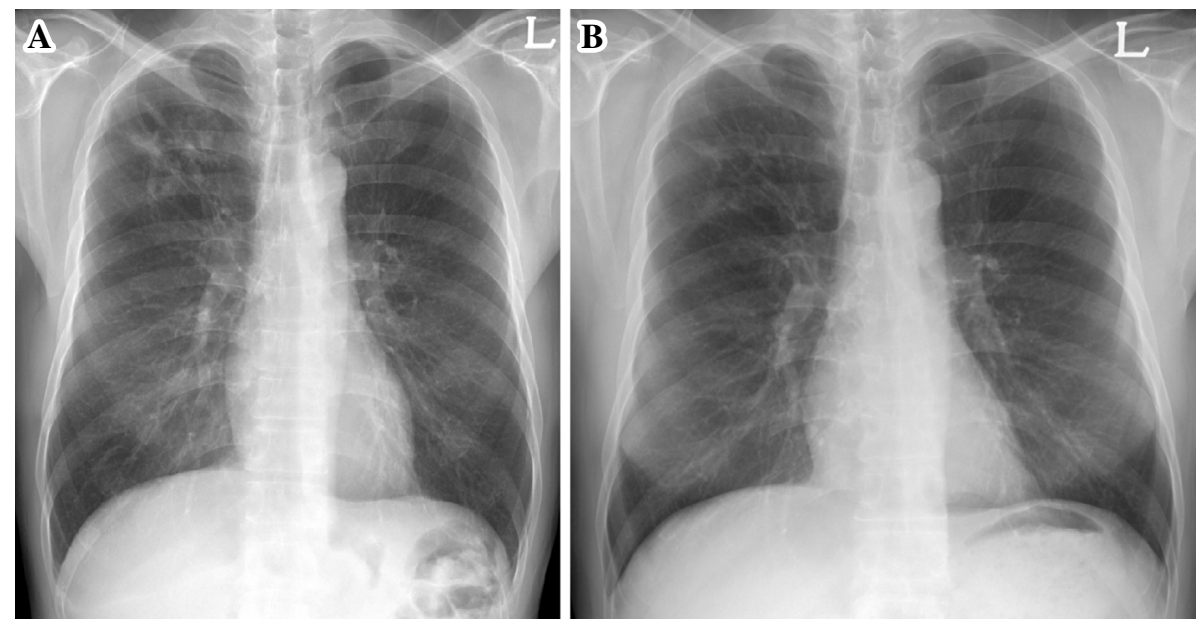

Figure 2. (A) Initial chest X-ray showing nodular and peribronchial increased opacity in the right upper lung field. (B) Chest X-ray at the time of treatment completion showing a decreased extent of nodular opacities in the right upper lung.

revealed INH-susceptible and RIF-resistant TB. The band pattern of the $r p o B$ gene of the Genotype ${ }^{\circledR}$ MTBDRplus assay was the same as that in the first case report, and DNA sequencing of the $r p o B$ gene revealed the same mutation of CTG511CCG. Based on this result and the elevated liver enzyme values, the regimen was transiently changed to INH, high-dose RIF (20 mg/kg) and EMB without PZA. Five weeks later, the phenotypic DST on Löwenstein-Jensen media showed the opposite result, with the culture now being resistant to INH and susceptible to RIF. An M. tuberculosis isolate was also resistant to $1 \mu \mathrm{g} / \mathrm{mL}$ of INH. Based on the results of phenotypic and genotypic DSTs, a final regimen of moxifloxacin, high-dose RIF $(20 \mathrm{mg} / \mathrm{kg})$ and EMB was initiated. DNA sequencing of the katG, inhA and oxyR-ahpC genes revealed that the three genes were wild-type.

The patient's symptoms improved rapidly after the initiation of the regimen including high-dose RIF, and culture conversion was seen at one month after the final regimen was initiated. Treatment was completed without any adverse events after a total of 10 months on the final regimen (Fig. 2B). The patient has been regularly followed up for 36 months since treatment completion without recurrence of TB.

\section{Discussion}

The $r p o B$ gene mutations of the 81-bp RRDR are detected in approximately $97 \%$ of RIF-resistant M. tuberculosis isolates (1). Among these mutations, the most commonly detected were GAC516GTC (D516V), CAC526TAC (H526Y), CAC526GAC (H526D) and TCG531TTG (S531L), all of which are included as mutation probes in the MTBDRplus assay. While these four mutations cause high-level RIF resistance (D516V mutation causes a RIF-resistant but rifabutin-susceptible phenotype), some rpoB mutations known as disputed mutations can cause low-level RIF resistance, defined as a minimal inhibitory concentration (MIC) of $0.063-0.5 \mu \mathrm{g} / \mathrm{mL}$ for RIF using the $7 \mathrm{H} 9$ Middlebrook medium (5). As this mutation can induce low-level RIF resistance, results can be discordant between phenotypic and genotypic susceptibility tests, e.g. the phenotypic DST may indicate susceptibility to RIF despite genotypic resistance to RIF. The lack of MIC data is a limitation of this study.

It has been reported that disputed mutations comprise approximately $10 \%$ of all rpoB mutations $(5,6)$. Along with the CTG511CCG mutation, which presented in both cases in the present study, the GAC516TAC (D516Y), CAC526CTC (H526L) and CTG533CCG (L533P) mutations are commonly observed disputed $r р о B$ mutations reported in previous studies (2-4). However, not all disputed mutation show low-level RIF resistance. For instance, a phenotypic DST revealed that approximately half of the tested strains with the CTG511CCG mutation were RIF-resistant (6).

Pulmonary TB with strains exhibiting low-level RIF resistance presents a therapeutic challenge in terms of high-dose RIF-based treatment. Since Van Ingen et al. reported that the $20 \mathrm{mg} / \mathrm{kg}$ dose of RIF is likely to overcome an MIC of 1 $\mu \mathrm{g} / \mathrm{mL}$ determined using $7 \mathrm{H} 10$ agar dilution methods for the GAC516TAC mutation (2), it has been suggested that a high-dose RIF (20 mg/kg)-based regimen might be feasible for isolates with disputed mutations exhibiting low-level resistance $(2,4)$. To our knowledge, these are the first reported cases of pulmonary TB patients infected with strains that had a disputed $r p o B$ mutation treated with such a regimen showing a successful outcome without one-year recurrence.

Although we failed to measure the MIC of the two strains in the present study, we judged them to have low-level RIF resistance based on the discrepant results between the phenotypic and genotypic DST. Despite the World Health Organization guideline recommending that any patient with RIF-resistant $\mathrm{TB}$ be treated with the recommended multidrug-resistant TB (MDR-TB) regimen (7), the cases in the present study received a simple regimen for a short pe- 
riod of time compared with the usual MDR-TB treatment. In addition, neither patient experienced any serious adverse events. Therefore, further studies involving more patients are needed to determine the efficacy of high-dose RIFcontaining regimens for treating cases of disputed rpoB mutations with low-level resistance to RIF.

In the first case, recurrent TB developed four months after treatment completion, suggesting that the TB was caused likely by a relapse rather than reinfection. Therefore, it is likely that the previous episode of pulmonary TB was also due to a $M$. tuberculosis isolate with a disputed $r p o B$ mutation. Considering that cases infected with M. tuberculosis isolates with disputed $r p o B$ mutations have had unfavorable outcomes when treated with the standard-dose RIF-based regimen even though the strains were found to be RIFsusceptible by a phenotypic DST $(5,8)$, the standard treatment for a disputed $r р о B$ mutation in the first case might have resulted in recurrence soon after the previous treatment had been completed. However, we were unable to confirm this possibility because we did not perform genotypic testing during the previous treatment period. This finding suggests that the use of both phenotypic and genotypic DST may be useful for optimizing the anti-TB treatment. The lack of genotypic data is another limitation of this study.

In summary, we herein report two cases of pulmonary TB patients with the disputed $r p o B$ mutation CTG511CCG that were successfully treated with a high-dose RIF-containing regimen.

The authors state that they have no Conflict of Interest (COI).

\section{References}

1. Telenti A, Imboden $P$, Marchesi $F$, et al. Detection of rifampicinresistance mutations in Mycobacterium tuberculosis. Lancet 341: 647-650, 1993.

2. Van Ingen J, Aarnoutse R, de Vries G, Boeree MJ, van Soolingen D. Low-level rifampicin-resistant Mycobacterium tuberculosis strains raise a new therapeutic challenge. Int $J$ Tuberc Lung Dis 15: 990-992, 2011

3. Van Deun A, Aung KJ, Hossain A, et al. Disputed rpoB mutations can frequently cause important rifampicin resistance among new tuberculosis patients. Int J Tuberc Lung Dis 19: 185-190, 2015.

4. Ho J, Jelfs P, Sintchencko V. Phenotypically occult multidrugresistant Mycobacterium tuberculosis: dilemmas in diagnosis and treatment. J Antimicrob Chemother 68: 2915-2920, 2013.

5. Ocheretina O, Escuyer VE, Mabou MM, et al. Correlation between genotypic and phenotypic testing for resistance to rifampin in Mycobacterium tuberculosis clinical isolates in Haiti: investigation of cases with discrepant susceptibility results. PLoS One 9: e90569, 2014.

6. Van Deun A, Aung KJ, Bola V, et al. Rifampin drug resistance tests for tuberculosis: challenging the gold standard. J Clin Microbiol 51: 2633-2640, 2013.

7. WHO treatment guidelines for drug-resistant tuberculosis, 2016 update [Internet]. [cited 2017 May. 15]. Available from: http://ww w.who.int/tb/areas-of-work/drug-resistant-tb/treatment/resources/en/

8. Williamson DA, Roberts SA, Bower JE, et al. Clinical failures associated with $r p o B$ mutations in phenotypically occult multidrugresistant Mycobacterium tuberculosis. Int J Tuberc Lung Dis 16: 216-220, 2012.

The Internal Medicine is an Open Access journal distributed under the Creative Commons Attribution-NonCommercial-NoDerivatives 4.0 International License. To view the details of this license, please visit (https://creativecommons.org/licenses/ by-nc-nd/4.0/).

(C) 2018 The Japanese Society of Internal Medicine Intern Med 57: 3281-3284, 2018 